\title{
Editorial
}

\section{Health care beyond professional health systems: The sociocultural context}

In many instances, patients do not follow medical advice as expected by medical professionals. There may be many reasons for not heeding such advice. Sometimes, patients follow more than one treatment method such as traditional "folk" practices and/or religious practices to improve the health condition. When a person contracts a disease or has symptoms of a disease, several steps are followed. First, the patient perceives and experiences symptoms. Then he/she and other members of the family, relatives and friends, etc. (non - medical professionals) label and evaluate symptoms. Sociocultural beliefs, values, cultural aspects and knowledge about illness in the community influence this process. Then the patient and others around decide "what to do". The decision includes whether to go to a medical professional or not, if so which system of medicine, which institution, which medical professional in the case of the private sector, and when to go. They may have taken several possible alternative healing methods even before approaching a professional medical practitioner. Therefore, engaging in specific health care seeking behavior is decided originally to a large extent by lay people. If they decide to seek a treatment from a health professional, then they will receive advice and treatment based on the scientific approach of the medical professional. Then the patient again goes back to his/her own community which will evaluate the treatment and the medical advice given. This evaluation and expressions by lay people highly influence the patient heeding medical advice, and/or seeking advice from another health professional or alternative healing method.

The communication between the ill person and the medical professional is not a simple encounter. Health seeking behavior and adherence to medical advice are influenced by many factors beyond professional medical services. Understanding these complex social interactions in the community is important to promote the appropriate health seeking behavior. These complex social interactions are equally important in preventive health care interventions as well. In many instances, health advice given by public health staff is not practiced completely by communities. For example, advice on feeding practices given by the Public Health Midwife may not be practiced by the mother, simply because of the influence of lay-people around her. Professor Arthur Kleinman, a physician and anthropologist extensively discusses health care in the social and cultural context. He identified three main sectors (i.e., popular sector, professional sector, folk sector) that prevail in any society which is important in influencing health related behaviours and activities of a person.

\section{Popular sector}

The popular sector represents the individual's family, neighbours, relatives, social networks, and community. Their beliefs and activities are the first to influence a person having an illness. The popular sector defines the illness, and functions as the chief source and most immediate determinant of care. Based on this determination, the decision is made whether to seek the assistance of the professional sector. If so, lay people seek health care by deciding when and whom to consult, whether or not to comply, when to 
switch between treatment alternatives, whether care is effective, and whether they are satisfied with its quality. Heeding to advice given by health professionals as well as medical treatment is depended on the popular sectors decisions. Popular sector forms the boundaries between the different sectors - it determines the points of entrance into, exit from, and interaction between the different sectors.

\section{Professional sector}

The professional sector comprises organized healing professions. In the Sri Lankan context, Western medicine dominates in both the curative and preventive health sector, while professional Ayurveda and other medical streams are also present. Each medical discipline creates its own clinical reality. In many instances, professional sector ignores the sociological aspects. Professional sectors attempt to define the problems and their solutions by theories they learn through their formal medical education; professional sanctioned solution frames are offered. Western medical practitioners attempt to explain and offer the solutions through biomedical science, while Ayurveda practitioners attempt to explain and offer the solutions through historically documented theories. The high profile academic knowledge considers patients, lay public and other professional health streams and "folk" practitioners as irrational and unscientific. Health professionals are usually insensitive to the views of clinical realities held by other healers (medical professionals of other medical disciplines) and to the expectations and beliefs of patients. Sometimes, this conflict affects the doctor-patient relationship as well.

\section{The folk sector}

The folk sector is a mixture of many different components; most are related to popular sector. Few examples of the "folk" sector includes manthras, rituals, some religious activities, blessings, etc. When the professional sector is lacking or inaccessible, the folk sector and popular sector constitute a large part of the health care system. In other words, the "folk" sector is more prominent, when health professionals are not easily accessible.

There may be simultaneous use of several healing methods such as traditional methods and "folk" healing methods in addition to taking the professional medical advice. As an example, a patients with diabetes may take the prescribed drugs at home as adviced by the Western medical doctor (sometimes they will not take the drugs regularly due to the influence from lay people), and at the same time he or she may try different remedies which are known through the popular sector, and may get blessings of "folk" sector. If there is any adverse effect, he or she will seek advice from the professional sector.

In the case of public health services, understanding of the "role" the "popular sector" and the "folk sector" is essential in planning health education and behavior change interventions.

\section{Sumal Nandasena}

Co-Editor

\section{Upul Senarath}

Editor

Upul Senarath, Editor/JCCPSL upul@commed.cmb.ac.lk

Sumal Nandasena, Co-Editor/JCCPSL sumalnandasena@gmail.com

Editorial Office email: editorjccps1@gmail.com

DOI: http://dx.doi.org/10.4038/jccpsl.v20i1.8066 